\title{
DEVELOPMENT OF JAPONICA TYPE CYTOPLASMIC MALE STERILE (CMS) RICE LINES FOR COMMERCIAL HYBRID RICE IN MEDITERRANEAN ECOLOGICAL CONDITION
}

\author{
Rasim UNAN \\ Black Sea Agricultural Research Institute, Samsun, TURKEY \\ *Corresponding author: rasimunan@hotmail.com
}

Received: 25.11.2019

\begin{abstract}
Hybrid rice has an average $15 \%$ to $20 \%$ yield advantage over inbred lines. The accessibility of stable cytoplasmic male sterility system (CMS) is vital for commercial hybrid rice. The experiment was conducted to develop japonica type CMS lines of national rice varieties. National varieties were tested to determine as maintainer, CMS or restorer features via test cross. The test crosses were made by using 19 japonica type national rice varieties and 21 CMS lines and 1 restorer line. All national rice varieties which tested are determined maintainer features. In addition, $8 \mathrm{CMS}$ introduction lines and 1 restorer lines adapted Mediterranean ecological condition for hybrid breeding process. Two maintainer were backcrossed for 6 generations and it was developed similar agronomic features and $100 \%$ spikelet sterile CMS lines. For production of CMS lines Osmancik-97 and Kiziltan, it was employed wild abortive rice which is CMS V20A. Successive backcrossing of varieties succeeded to produce completely male sterile CMS lines. Wild abortive cytoplasm did not affect agronomic characteristic. Osmancik-97 and Kiziltan were utilized as a recurrent parent in breeding program due to their popularity, and the new WA-CMS lines might be useful for hybrid rice breeding. CMS-Osmancik-97 and CMS-Kiziltan are the first developed national CMS rice lines in Turkey.
\end{abstract}

Keywords: Breeding, cytoplasmic male sterility (CMS), hybrid rice, Oryza sativa, Osmancik-97

\section{INTRODUCTION}

It has been calculated that hybrid rice provides $15-20 \%$ yield advantage over conventional rice. The country with the highest hybrid rice production area compared to conventional rice area is China with $56 \%$. After that, the countries with the highest hybrid production area are the USA, Vietnam, Bangladesh, Philippines, India, and Indonesia with $14.5 \%, 9.4,6.8 \%, 4.3 \%, 3.2 \%$, and $0.5 \%$, respectively (Barclay, 2010). Hybrids have been used efficaciously especially far East since 1975 however it is new tool for Europe. Initial step is to supply the male sterile (MS) lines to hybrid breeding framework. Genetic and non-genetic mechanisms are available to exploit for male sterility. Hybrid rice is produced by two or three lines breeding methods. Two lines method included thermo-sensitive genic male sterile line (TGMS) and Chemical Hybridizing Agents (CHA). Our previous studies focused on determine the better method for Mediterranean ecological condition. Sterility of male is specified $22-49 \%$ when sprayed gametocides in some cultivars. TGMS lines which TGMS1 and TGMS2 have $89-96 \%$ sterility in the uncontrolled field circumstance. CMS lines have been determined $100 \%$ male sterility (Unan et al., 2016). It was revealed that three lines breeding method is more stable for Mediterranean ecological condition. And also the most of hybrid rice varieties have been released subject to 3-line system, including $\mathrm{R}$ (restorer line), A (CMS line), and B (maintainer line). Three-line system utilize CMS lines especially wild abortive type CMS (WA-CMS) lines (Sattari et al., 2007).

Utilization of CMS framework might be fitting methodology for tradal hybrid rice. CMS frameworks are constrained by related of cytoplasm with nucleus gene (Kaul, 1997). Advancement of commercial hybrids utilizing CMS need a restorer R-line, a male sterile A-line, and a maintainer B-line. Principle attributes of these lines are A-line is male sterile, which are utilized as female line hybrid breed multiplying plot. Near-isogenic lines of Aline is B-line, which are expected to maintain A-line. Bline is considered as key line without that A-line could not be kept up (Ali et al., 2014). Fruitful utilization of hybrid breeds vigor in rice to a greatly related to the accessibility of regional developed restorer and CMS lines (Kumar et al., 1996). It was assessed CMS lines from Chinese which Zhen Shan97A and V20A alongside their maintainer, but the lines were not adapted Bangladesh ecological condition because of high susceptibility to diseases and insects (Julfiquar et al., 2002). Besides, plant breeders need wide scope of CMS lines to deliver wanted hybrids. 
Consequently, it is important to move accessible CMS framework into local elite breeding lines. Thus, utilization of some CMS lines might be useful to ease the issue, and to create versatile, heterotic cross breeds (Ali et al., 2014).

The aim of this study is development of japonica type CMS lines for commercial hybrid breeding. In addition, second aim is to determine adaptable CMS-maintainer and restorer lines for Mediterranean ecological condition.

\section{MATERIALS AND METHODS}

These experiments were conducted in rice experimental fields and plant growth chamber of the Trakya Agricultural Research Institute located in Edirne, Turkey $\left(41^{\circ} 38^{\prime} 41^{\prime \prime} \mathrm{N}, 26^{\circ} 35^{\prime} 46^{\prime \prime} \mathrm{E}\right)$ between 2015 and 2019 during the summer season. Nineteen national japonica type rice variety, 1 restorer variety, $21 \mathrm{CMS}$ lines and their corresponding maintainer lines was formed the material for the current examination. National varieties, restorer variety, A-lines, and B-lines lines were planted in two rows per plots having 10 hills per row with spacing of $20 \times 20 \mathrm{~cm}$ apart. Field evaluation was conducted at experiment field, Edirne, Turkey between 2015-2019. Osmancik-97 and Kiziltan varieties are among the famous Turkish varieties (Tatar and Gevrek, 2007; Gevrek, 2012) so they were utilized a recurrent parent for backcross process.

Spikelet fertility $(\%)=($ Filled grains per panicle $/$ Total spikelet per panicle) $\times 100$

Spikelet sterility $(\%)=($ Non-filled grains per panicle $/$ Total spikelet per panicle) $\times 100$

Observations were recorded on five randomly selected panicle isolated plants of each lines in each replication for various morphological and floral traits. Pedigree backcross method was utilized to breeding progress. The agronomic observation was evaluated by Standard Evaluation System for Rice (IRRI, 2014). Data were analyzed using analysis of variance (SAS, 2002) and means of treatment, and were compared based on the least significant difference test (LSD) at a 0.05 probability level.

\section{RESULTS AND DISCUSSION}

\section{Evaluation of CMS lines for Mediterranean ecological condition}

Currently developed and designated CMS lines and their maintainers were evaluated as CMS source in lowland ecology (IRRI, 1997). In Mediterranean ecological conditions, CMS lines having characteristic like early or moderate $50 \%$ flowering time and maturity, short height good panicle length. Twenty-one CMS and their maintainer lines were evaluated according to some agronomical characters both 2015 and 2016. V20A, 15A, 23A, 31A, 76A, IR93559A, IR93560A, and IR93563A were classified suitable in most of characteristics such as early flowering time, short height, and panicle length (Table 1). Flowering time observation revealed that early, moderate and, late flowering days, as 80-84 days, 86-88 days and, 98-135 days, respectively. Plant length ranged between $48-108 \mathrm{~cm}$ and, panicle length was between $13-16 \mathrm{~cm}$. Result showed that existence of significant differences amongst lines for all the characteristics assessed. In different investigation significant varying was noted amongst the distinctive class or lines researched by Jain et al. (2017).

\section{Identification of maintainer and restorer lines against} V20A

It is the breeding nursery which F1s of cytoplasmic male sterile lines and test varieties from the source nursery are screened for spikelet fertility, and other agronomic characteristic to classify the possible restorers, maintainers, and heterotic hybrids (IRRI, 1997). Spikelet fertility of $\mathrm{F}_{1}$ s between $V 20 A$ and 19 national rice and, $\mathrm{F}_{1}$ between $V 20 A$ and one restorer line (IR50) as a control were determined during of 2015-2016 for classification of restorer and maintainer lines. Spikelet sterility was $100 \%$ for all $\mathrm{F}_{1} \mathrm{~s}(V 20 A \times 19$ National Rice $)$ and spikelet fertility was 90.6 for restorer control cross $(V 20 A \times I R 50)$. Considering the results of two years, $19 \quad F_{1} s$ were designated as completely sterile and it was estimated that the pollen parents carry maintainer genes. The 19 national rice varieties may be specified as maintainer lines against CMS lines (Table 2). The result of this experiment are somehow higher than the finding of Govinda Raj and Virmani (1989). Their results are as $24 \%$ restorer and $40 \%$ maintainer when tested 37 indica/japonica derivatives against CMS. And also our results are higher than results of Ali et al. (2014), they found 23 out of 129 crosses (15\%) contributed straightforwardly to recognize restorers and maintainers. This distinction may be because of the hereditary foundation of the assessed genotypes.

\section{Backcross breeding nursery}

Backcross nursery is wherein the CMS framework from the accessible CMS lines is moved into the hereditary background of elit maintainer lines distinguished in the test-cross nursery by continuous backcrossing (IRRI, 1997). Test-cross is first cross between elite lines and CMS lines of an ideal cytosterility source to test their maintaining capacity. These elite lines which are classified as maintainer lines are repeatedly backcrossed up to six generations for complete transfer of cytosterility source (Figure 1). Osmancik-97 and Kiziltan japonica varieties was successfully backcrossed with CMS $V 20 A$ for six generations (Table 3). The similar results received that the other studies that the transfer by backcrossing into elite lines of the cytosterility system of CMS wild abortive (WA) lines Er-Jiu-Nan 1A, V20A, V41A, and Zhen Shan 97A (Yuan and Virmani, 1988; Leon et al., 1997). 
Table 1. Evaluation of agronomic traits and adaptability of CMS lines in Mediterranean Ecological Condition in 2015 and 2016

\begin{tabular}{|c|c|c|c|c|c|}
\hline Order & CMS and Maintainer Lines & $\begin{array}{l}\text { Flowering Days } \\
\text { (Day) }\end{array}$ & Flowering Days Situation & $\begin{array}{l}\text { Plant Length } \\
\text { (cm) }\end{array}$ & $\begin{array}{l}\text { Panicle Length } \\
\text { (cm) }\end{array}$ \\
\hline 1 & V20A & $81 \mathrm{w}$ & Early & $51.2 \mathrm{w}$ & $14.6 \mathrm{~m}$ \\
\hline 2 & V20B & $80 x$ & Early & $52.8 \mathrm{w}$ & $14.8 \mathrm{~lm}$ \\
\hline 3 & $15 \mathrm{~A}$ & $83 \mathrm{t}$ & Early & $53.6 \mathrm{w}$ & $15.6 \mathrm{jm}$ \\
\hline 4 & $15 B$ & $82 \mathrm{u}$ & Early & $54.8 \mathrm{w}$ & $15.8 \mathrm{im}$ \\
\hline 5 & $31 \mathrm{~A}$ & $88 \mathrm{q}$ & Moderate & $72.2 \mathrm{im}$ & 20.4 ai \\
\hline 6 & $31 \mathrm{~B}$ & $86 \mathrm{~s}$ & Moderate & $73.4 \mathrm{hl}$ & $21.0 \mathrm{ag}$ \\
\hline 7 & $23 \mathrm{~A}$ & $87 \mathrm{r}$ & Moderate & $65,6 \mathrm{qv}$ & $16,6 \mathrm{gm}$ \\
\hline 8 & $23 \mathrm{~B}$ & $86 \mathrm{~s}$ & Moderate & $66.8 \mathrm{ou}$ & $17.4 \mathrm{fm}$ \\
\hline 9 & $76 \mathrm{~A}$ & $83 \mathrm{u}$ & Early & $61,8 \mathrm{v}$ & $18,2 \mathrm{dm}$ \\
\hline 10 & $76 \mathrm{~B}$ & $81 \mathrm{w}$ & Early & $63.0 \mathrm{tv}$ & $18.8 \mathrm{~cm}$ \\
\hline 11 & IR58025A & $135 \mathrm{a}$ & Very Late & $64,0 \mathrm{sv}$ & $17,4 \mathrm{fm}$ \\
\hline 12 & IR58025B & $132 \mathrm{~b}$ & Very Late & $65,2 \mathrm{rv}$ & $18.4 \mathrm{dm}$ \\
\hline 13 & IR62829A & 1201 & Very Late & $69.8 \mathrm{lp}$ & $18.0 \mathrm{em}$ \\
\hline 14 & IR62829B & $117 \mathrm{n}$ & Very Late & $71.0 \mathrm{jn}$ & $18.8 \mathrm{~cm}$ \\
\hline 15 & IR68886A & $121 \mathrm{k}$ & Very Late & $69.6 \mathrm{lq}$ & $19.8 \mathrm{ak}$ \\
\hline 16 & IR68886B & $117 \mathrm{n}$ & Very Late & $70.8 \mathrm{ko}$ & $20.6 \mathrm{ah}$ \\
\hline 17 & IR69625A & $125 \mathrm{~g}$ & Very Late & $68.6 \mathrm{mr}$ & $19.4 \mathrm{al}$ \\
\hline 18 & IR69625B & $123 \mathrm{i}$ & Very Late & $69.8 \mathrm{lp}$ & $20.2 \mathrm{aj}$ \\
\hline 19 & IR73793A & $124 \mathrm{~h}$ & Very Late & $79.0 \mathrm{cf}$ & $23.6 \mathrm{ab}$ \\
\hline 20 & IR73793B & $122 \mathrm{j}$ & Very Late & $80.2 \mathrm{~cd}$ & $24.4 \mathrm{a}$ \\
\hline 21 & IR75596A & 1201 & Very Late & $75.4 \mathrm{fi}$ & 21.4 af \\
\hline 22 & IR75596B & $118 \mathrm{~m}$ & Very Late & $76.6 \mathrm{dh}$ & $22.4 \mathrm{ae}$ \\
\hline 23 & IR75606A & $127 \mathrm{e}$ & Very Late & $85.2 \mathrm{ab}$ & $22.4 \mathrm{ae}$ \\
\hline 24 & IR75606B & $125 \mathrm{~g}$ & Very Late & $86.4 \mathrm{a}$ & $23.4 \mathrm{ac}$ \\
\hline 25 & IR78369A & $126 \mathrm{f}$ & Very Late & $75.0 \mathrm{fj}$ & $19.0 \mathrm{bm}$ \\
\hline 26 & IR78369B & $124 \mathrm{~h}$ & Very Late & 75.8 ei & $19.6 \mathrm{ak}$ \\
\hline 27 & IR79156A & $127 \mathrm{e}$ & Very Late & $78.6 \mathrm{cg}$ & 21.6 af \\
\hline 28 & IR79156B & $125 \mathrm{i}$ & Very Late & $79.8 \mathrm{ce}$ & $22.8 \mathrm{ad}$ \\
\hline 29 & IR80151A & $130 \mathrm{c}$ & Very Late & $63.2 \mathrm{tv}$ & $15.8 \mathrm{im}$ \\
\hline 30 & IR80151B & $128 \mathrm{~d}$ & Very Late & $64.4 \mathrm{sv}$ & $17.0 \mathrm{fm}$ \\
\hline 31 & IR80156A & $126 \mathrm{f}$ & Very Late & $73.4 \mathrm{hl}$ & $19.0 \mathrm{bm}$ \\
\hline 32 & IR80156B & $123 \mathrm{~g}$ & Very Late & $74.8 \mathrm{gk}$ & $20.2 \mathrm{aj}$ \\
\hline 33 & IR80564A & $123 \mathrm{i}$ & Very Late & 81.2 bc & 20.4 ai \\
\hline 34 & IR80564B & 1201 & Very Late & $82.4 \mathrm{ac}$ & 21.4 af \\
\hline 35 & IR93559A & $84 \mathrm{~s}$ & Early & $62.8 \mathrm{uv}$ & $15.4 \mathrm{~km}$ \\
\hline 36 & IR93559B & $82 \mathrm{v}$ & Early & $64.0 \mathrm{sv}$ & $16.6 \mathrm{gm}$ \\
\hline 37 & IR93560A & $81 \mathrm{w}$ & Early & $63.0 \mathrm{tv}$ & $16.0 \mathrm{hm}$ \\
\hline 38 & IR93560B & $80 x$ & Early & $64.2 \mathrm{sv}$ & $17.2 \mathrm{fm}$ \\
\hline 39 & IR93562A & $100 \mathrm{o}$ & Late & $65.0 \mathrm{rv}$ & $17.6 \mathrm{fm}$ \\
\hline 40 & IR93562B & $98 \mathrm{p}$ & Late & $66.4 \mathrm{pu}$ & $19.0 \mathrm{bm}$ \\
\hline 41 & IR93563A & $88 \mathrm{q}$ & Moderate & $67.0 \mathrm{nt}$ & $18.8 \mathrm{~cm}$ \\
\hline 42 & IR93563B & $86 \mathrm{~s}$ & Moderate & $67.8 \mathrm{~ns}$ & $19.6 \mathrm{bk}$ \\
\hline \multicolumn{2}{|c|}{ F Ratio of lines } & $12160.3 * *$ & & $34.4 * *$ & $8.7 * *$ \\
\hline \multicolumn{2}{|c|}{$\mathrm{CV} \%$} & 0.4 & & 4.6 & 9.9 \\
\hline \multicolumn{2}{|l|}{ LSD } & 0.5 & & 4.0 & 4.8 \\
\hline
\end{tabular}

*: $\mathrm{P}<0.05, * *: \mathrm{P}<0.01, \mathrm{~ns}$ : nonsignificant, values followed by the same letter are not statistically significantly different. 
Table 2. Sterility maintenance of 19 national rice varieties and fertility restoration ability one restorer rice against V20A CMS line, in 2015 and 2016, and status remarks

\begin{tabular}{|c|c|c|c|c|}
\hline Variety Name & Type & $\begin{array}{l}\text { Sterility } \\
(\%) 2015\end{array}$ & $\begin{array}{l}\text { Sterility } \\
(\%) 2016\end{array}$ & Status \\
\hline IR50 & Indica & $9.0 \mathrm{hi}$ & $9.9 \mathrm{~g}$ & Restorer \\
\hline V20A & Wild Abortive & $100 \mathrm{a}$ & $100 \mathrm{a}$ & CMS \\
\hline $\mathrm{V} 20 \mathrm{~A} \times \operatorname{IR} 50\left(\mathrm{~F}_{1}\right)$ & Indica & $9.2 \mathrm{gi}$ & $10.1 \mathrm{f}$ & Hybrid \\
\hline Osmancik-97 × V20A (BC3 and BC4) & Japonica & $100 \mathrm{a}$ & $100 \mathrm{a}$ & CMS \\
\hline Osmancik-97 & Japonica & $9.3 \mathrm{fh}$ & $9.6 \mathrm{I}$ & Maintainer \\
\hline V20A $\times$ Kiziltan $(B C 3$ and BC4 $)$ & Japonica & $100 \mathrm{a}$ & $100 \mathrm{a}$ & CMS \\
\hline Kiziltan & Japonica & $8.8 \mathrm{ij}$ & $8.8 \mathrm{j}$ & Maintainer \\
\hline V20A $\times$ Halilbey $(B C 2$ and BC3 $)$ & Japonica & $100 \mathrm{a}$ & $100 \mathrm{a}$ & CMS \\
\hline Halilbey & Japonica & $9.3 \mathrm{fh}$ & 9.7 hi & Maintainer \\
\hline V20A $\times$ Tosyagunesi $(\mathrm{BC} 2$ and $\mathrm{BC} 3)$ & Japonica & $100 \mathrm{a}$ & $100 \mathrm{a}$ & CMS \\
\hline Tosyagunesi & Japonica & $8.5 \mathrm{j}$ & $6.8 \mathrm{k}$ & Maintainer \\
\hline $\mathrm{V} 20 \mathrm{~A} \times$ Gonen $(\mathrm{BC} 2$ and $\mathrm{BC} 3)$ & Japonica & $100 \mathrm{a}$ & $100 \mathrm{a}$ & CMS \\
\hline Gonen & Japonica & 6.31 & 9.7 hi & Maintainer \\
\hline $\mathrm{V} 20 \mathrm{~A} \times \operatorname{Ece}(\mathrm{BC} 2$ and $\mathrm{BC} 3)$ & Japonica & $100 \mathrm{a}$ & $100 \mathrm{a}$ & CMS \\
\hline Ece & Japonica & $10.1 \mathrm{c}$ & $11.6 \mathrm{e}$ & Maintainer \\
\hline V20A $\times$ Pasali $(B C 2$ and $B C 3)$ & Japonica & $100 \mathrm{a}$ & $100 \mathrm{a}$ & CMS \\
\hline Pasali & Japonica & $6.3 \mathrm{~m}$ & $9.8 \mathrm{gh}$ & Maintainer \\
\hline $\mathrm{V} 20 \mathrm{~A} \times \mathrm{Beser}(\mathrm{BC} 2$ and $\mathrm{BC} 3)$ & Japonica & $100 \mathrm{a}$ & $100 \mathrm{a}$ & CMS \\
\hline Beser & Japonica & $9.7 \mathrm{de}$ & $10.1 \mathrm{f}$ & Maintainer \\
\hline $\mathrm{V} 20 \mathrm{~A} \times$ Edirne $(\mathrm{BC} 2$ and $\mathrm{BC} 3)$ & Japonica & $100 \mathrm{a}$ & $100 \mathrm{a}$ & CMS \\
\hline Edirne & Japonica & 6.31 & $9.8 \mathrm{gh}$ & Maintainer \\
\hline V20A $\times$ Duragan $(B C 2$ and BC3) & Japonica & $100 \mathrm{a}$ & $100 \mathrm{a}$ & CMS \\
\hline Duragan & Japonica & $9.4 \mathrm{eg}$ & $12.7 \mathrm{c}$ & Maintainer \\
\hline $\mathrm{V} 20 \mathrm{~A} \times$ Kale $(\mathrm{BC} 2$ and $\mathrm{BC} 3)$ & Japonica & $100 \mathrm{a}$ & $100 \mathrm{a}$ & CMS \\
\hline Kale & Japonica & $7.8 \mathrm{k}$ & $8.7 \mathrm{j}$ & Maintainer \\
\hline V20A $\times$ IMIHalilbey $(\mathrm{BC} 2$ and BC3) & Japonica & $100 \mathrm{a}$ & $100 \mathrm{a}$ & CMS \\
\hline IMIHalilbey & Japonica & $10.1 \mathrm{c}$ & $12.1 \mathrm{~d}$ & Maintainer \\
\hline V20A $\times$ IMIOsmancik (BC2 and BC3) & Japonica & $100 \mathrm{a}$ & $100 \mathrm{a}$ & CMS \\
\hline IMIOsmancik & Japonica & $9.8 \mathrm{~cd}$ & $10.2 \mathrm{f}$ & Maintainer \\
\hline V20A $\times$ Hamzadere $(\mathrm{BC} 2$ and $\mathrm{BC} 3)$ & Japonica & $100 \mathrm{a}$ & $100 \mathrm{a}$ & CMS \\
\hline Hamzadere & Japonica & $7.7 \mathrm{k}$ & $8.7 \mathrm{j}$ & Maintainer \\
\hline V20A $\times$ HalilbeyPi40 $(\mathrm{BC} 2$ and BC3) & Japonica & $100 \mathrm{a}$ & $100 \mathrm{a}$ & CMS \\
\hline HalilbeyPi40 & Japonica & $9.5 \mathrm{df}$ & $9.9 \mathrm{~g}$ & Maintainer \\
\hline $\mathrm{V} 20 \mathrm{~A} \times$ OsmancikPi40 $(\mathrm{BC} 2$ and $\mathrm{BC} 3)$ & Japonica & $100 \mathrm{a}$ & $100 \mathrm{a}$ & CMS \\
\hline OsmancikPi40 & Japonica & $9.5 \mathrm{df}$ & $9.8 \mathrm{gh}$ & Maintainer \\
\hline $\mathrm{V} 20 \mathrm{~A} \times$ Tunca $(\mathrm{BC} 2$ and $\mathrm{BC} 3)$ & Japonica & $100 \mathrm{a}$ & $100 \mathrm{a}$ & CMS \\
\hline Tunca & Japonica & $18.9 \mathrm{~b}$ & $20.5 \mathrm{~b}$ & Maintainer \\
\hline $\mathrm{V} 20 \mathrm{~A} \times$ Yildiz $(\mathrm{BC} 1$ and $\mathrm{BC} 2)$ & Japonica & $100 \mathrm{a}$ & $100 \mathrm{a}$ & CMS \\
\hline Yildiz & Japonica & NA & NA & Maintainer \\
\hline V20A $\times$ Boyabatkalesi $(\mathrm{BC} 1$ and $\mathrm{BC} 2)$ & Japonica & $100 \mathrm{a}$ & $100 \mathrm{a}$ & CMS \\
\hline Boyabatkalesi & Japonica & NA & NA & Maintainer \\
\hline F Ratio of lines & & $143682 * *$ & $1573390 * *$ & \\
\hline $\mathrm{CV}(\%)$ & & 0.4 & 0.1 & \\
\hline LSD & & 0.4 & 0.1 & \\
\hline
\end{tabular}




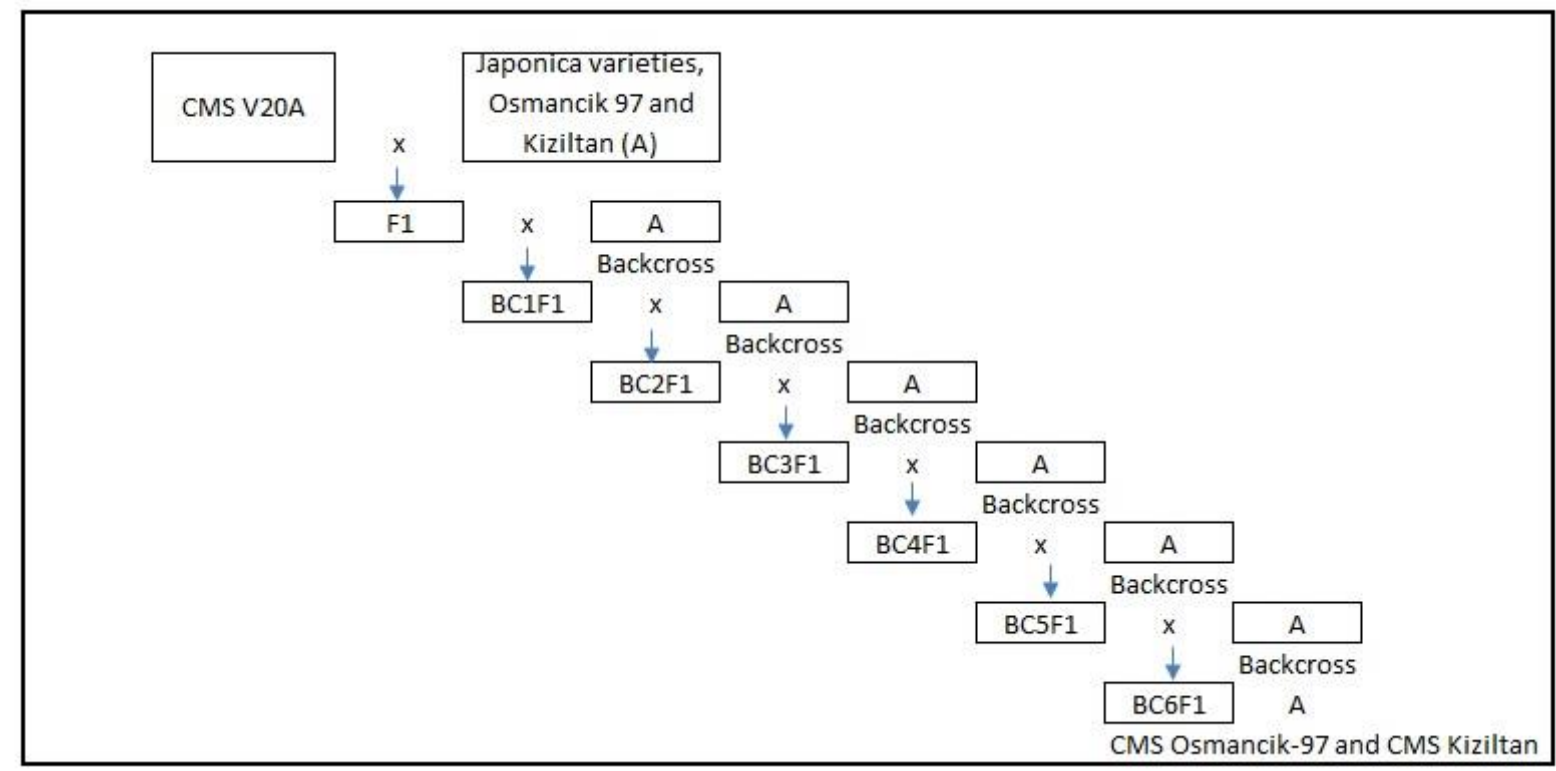

Figure 1. Flowchart of breeding method of new CMS lines

Table 3. Seed setting rates (\%) of backcrossed lines and one hybrid in 2017 and 2018

\begin{tabular}{|c|c|c|c|c|c|}
\hline \multirow{2}{*}{$\begin{array}{l}\text { Initial } \\
\text { Female } \\
\text { Parent }\end{array}$} & \multirow[b]{2}{*}{$\begin{array}{l}\text { Recurrent Polen } \\
\text { Parent }\end{array}$} & \multicolumn{2}{|c|}{2017} & \multicolumn{2}{|c|}{2018} \\
\hline & & Backcross & $\begin{array}{l}\text { Spikelet } \\
\text { Fertility }^{\text {a }}(\%)\end{array}$ & Backcross & $\begin{array}{l}\text { Spikelet Fertility } \\
(\%)\end{array}$ \\
\hline V20A & Osmancik-97 & $\mathrm{BC}_{5} \mathrm{~F}_{1}$ & $0.0 \mathrm{~b}$ & $\mathrm{BC}_{6} \mathrm{~F}_{1}$ & $0.0 \mathrm{~b}$ \\
\hline V20A & Kiziltan & $\mathrm{BC}_{5} \mathrm{~F}_{1}$ & $0.0 \mathrm{~b}$ & $\mathrm{BC}_{6} \mathrm{~F}_{1}$ & $0.0 \mathrm{~b}$ \\
\hline $31 \mathrm{~A}$ & Osmancik-97 & $\mathrm{BC}_{5} \mathrm{~F}_{1}$ & $0.0 \mathrm{~b}$ & $\mathrm{BC}_{6} \mathrm{~F}_{1}$ & $0.0 \mathrm{~b}$ \\
\hline $31 \mathrm{~A}$ & Kiziltan & $\mathrm{BC}_{5} \mathrm{~F}_{1}$ & $0.0 \mathrm{~b}$ & $\mathrm{BC}_{6} \mathrm{~F}_{1}$ & $0.0 \mathrm{~b}$ \\
\hline V20A & IR50 (Restorer) & $\mathrm{F}_{1}$ & $90.3 \mathrm{a}$ & $\mathrm{F}_{1}$ & $91.2 \mathrm{a}$ \\
\hline \multicolumn{2}{|c|}{ F Ratio of lines } & & $6115 * *$ & & $2772 * *$ \\
\hline \multicolumn{2}{|l|}{$\mathrm{CV}(\%)$} & & 4.94 & & 7.44 \\
\hline \multicolumn{2}{|l|}{ LSD } & & 1.68 & & 2.51 \\
\hline
\end{tabular}

${ }^{a}$ Average spikelet fertility of three plants, each containing three bagged panicles. *: $\mathrm{P}<0.05$, **: $\mathrm{P}<0.01$, ns: nonsignificant, values followed by the same letter are not statistically significantly different.

Agronomic characteristics were compared among Osmancik-97, CMS-Osmancik-97, Kiziltan, and CMSKiziltan in 2019. The experiment plants were transferred in the rice field at a dispersing of $20 \mathrm{~cm}$ between plants inside columns and $20 \mathrm{~cm}$ between lines. It was not found significant differences between the CMS lines and their maintainer lines in plant length, panicle length, flowering time and anthesis time (Table 4). National rice varieties had a similar agronomic features the other reports (Unan et al., 2013; Sezer et al., 2016)

Table 4. Comparison of some agronomic features between CMS lines and maintainer lines in 2019

\begin{tabular}{|c|c|c|c|c|c|c|c|c|c|c|}
\hline Properties & $\begin{array}{c}\text { Osmancik- } \\
97\end{array}$ & $\begin{array}{c}\text { CMS- } \\
\text { Osmancik- } \\
97\end{array}$ & F Ratio & $\begin{array}{l}\mathrm{CV} \\
(\%)\end{array}$ & LSD & Kiziltan & $\begin{array}{c}\text { CMS- } \\
\text { Kiziltan }\end{array}$ & F Ratio & $\begin{array}{l}\mathrm{CV} \\
(\%)\end{array}$ & LSD \\
\hline Plant Length $(\mathrm{cm})$ & 100.3 & 98.4 & 10.83 & 0.7 & $\mathrm{~ns}$ & 85.4 & 84.3 & 3.63 & 0.8 & $\mathrm{~ns}$ \\
\hline Panicle Length $(\mathrm{cm})$ & 16.4 & 16.2 & 4.92 & 0.9 & $\mathrm{~ns}$ & 15.3 & 15.2 & 2.28 & 0.7 & ns \\
\hline $\begin{array}{c}\text { Panicle Exsertion } \\
\text { rate }(\%)\end{array}$ & $100.0 \mathrm{a}$ & $86.4 \mathrm{~b}$ & $\begin{array}{c}13872 \\
* *\end{array}$ & 0.2 & 0.47 & 100.0 & 85.3 & $10282 * *$ & 0.2 & 0.60 \\
\hline $\begin{array}{l}\text { Flowering time } \\
\text { (day) }\end{array}$ & $85.0 \mathrm{~b}$ & $86.3 \mathrm{a}$ & 16.00 & 0.5 & ns & $83.3 b$ & $84.7 \mathrm{a}$ & 16.00 & 0.5 & ns \\
\hline Sterility (\%) & $9.5 b$ & $100.0 \mathrm{a}$ & $\begin{array}{c}98283 \\
* *\end{array}$ & 0.6 & 1.2 & $8.8 \mathrm{~b}$ & $100.0 \mathrm{a}$ & $6238 * *$ & 2.6 & 4.90 \\
\hline Anthesis time & $\begin{array}{l}11.30- \\
13.30\end{array}$ & $\begin{array}{l}11.30- \\
13.30\end{array}$ & _ & - & - & $\begin{array}{l}11.30- \\
13.30\end{array}$ & $\begin{array}{l}11.30- \\
13.30\end{array}$ & - & - & - \\
\hline
\end{tabular}

\footnotetext{
*: $\mathrm{P}<0.05, * *: \mathrm{P}<0.01$, ns: nonsignificant, values followed by the same letter are not statistically significantly different.
} 


\section{CONCLUSION}

It was developed japonica type CMS lines of Osmancik-97 and Kiziltan, which did not set any seeds in temperate climate region. The CMS-Osmancik-97 and $C M S$-Kiziltan might be useful for commercial hybrid rice breeding. Eight introduction source CMS lines (V20A, $15 A, \quad 23 A, \quad 31 A, \quad 76 A, \quad I R 93559 A, \quad I R 93560 A$, and $I R 93563 A)$ and one restorer line (IR 50) are useful for hybrid rice breeding programs ins Mediterranean ecological condition. New japonica type CMS lines have characteristics such as short height, early maturity, good panicle exsertion and panicle length which are a significant influence on commercial hybrid rice production.

\section{ACKNOWLEDGMENT}

This research was supported by Republic of Turkey, Ministry of Agriculture and Forestry, General Directorate of Agricultural Research and Policies (Project No: TAGEM/TBAD/16/A12/P04/001). This article selected in the Turkey 13th National and $1^{\text {st }}$ International Field Crops Conference in 2019.

\section{LITERATURE CITED}

Ali, M., M.A. Hossain, M.J. Hasan and M.E. Kabir. 2014. Identification of Maintainer And Restorer Lines In Local Aromatic Rice (Oryza Sativa). Bangladesh J. Agril. Res. 39(1): 1-12.

Barclay, A. 2010. Hybridizing the world. Rice Today 9:32-35.

Gevrek, M.N. 2012. Some Agronomic and Quality Characteristics of New Rice Varieties In The Aegean Region Of Turkey. Turkish Journal of Field Crops, 2012, 17(1):7477.

Govinda, R.K. and S.S. Virmani. 1989. Maintainer and restorers for different cytoplasmic male sterile systems. Intl. Rice Res. Newsl. 14(5): 7-8.

IRRI. 1997. Hybrid rice breeding manual. International rice research institute. Los Banos. Philippines.

IRRI. 2014. Standard evaluation system for rice (SES). 5th Eition, Los Banos, International Rice Research Institute. Philippines.

Jain, M., R. Kaur and S.S. Yadav. 2017. Phenotypic characterization of new cytoplasmic male sterile lines in rice (Oryza sativa L.). Journal of Pharmacognosy and Phytochemistry. 6(3): 166-167.
Julfiquar, A.W., J. M. Hasan, A.K. Azad and A.M. Nurunnabi. 2002. Research and development of hybrid rice in Bangladesh: A motivation to adoption. Proc. First National Workshop on "Research and Development of Hybrid Rice in Bangladesh, Progress and Future Strategies". Bangladesh Rice Res. Inst., held on 5-6 January. 2002. 9-19Pp.

Kaul, M.L.H. 1997. Male sterility: cytological, biological and molecular alterations. In: Plant Breeding and Crop Improvement. Vol. (II) (Eds. Kapoor and Saini) CBS Publishers Distribution. New Delhi. 377-378Pp.

Kumar, R.V., P.V. Satyanarayana and M.S. Rao.1996. New cytoplasmic male sterile lines developed in Andhra Pradesh, India. Intl. Rice Res. Notes 21(2-3): 30.

Leon, J.C., E.D. Redona, I.A. Cruz, M.F. Ablaza, F.M. Malabanan, R.J. Lara and S.R. Obien. 1997. Hybrid rice in the Philippines: progress and prospects. Advances in hybrid rice technology. Proceedings of the 3rd International Symposium on Hybrid Rice, 14-16 November 1996, Hyderabad, India. Manila (Philippines): International Rice Research Institute.

SAS. 2002. SAS Institute. SAS User's Guide: Statistics, v. 9.1.3. Cary, NC, USA: SAS Institute

Sattari, M., A. Kathiresan, G.B. Gregorio, J.E Hernandez, T.M. Nas and S.S. Virmani. 2007. Development and use of a twogene marker-aided selection system for fertility restorer genes in rice. Euphytica 153:35-42. doi:10. 1007/s10681006-9213-5.

Sezer, I., R. Unan, M. Sahin and M.O. Way. 2016. The effect of trinexapac-ethyl and seeding rate on rice milling yields. Turkish Journal of Agriculture and Forestry 40: 53-61 doi:10.3906/tar-1406-143.

Tatar, O. and M. Gevrek. 2007. Effects of Salt Stress on Some Physiolagical Characters of Rice (Oryza sativa L.) in Germination and Seedling Stages. Turkish Journal of Field Crops 12(1): 34-39.

Unan, R., I. Sezer, M. Sahin and L.A.J. Mur. 2013. Control of lodging and reduction in plant length in rice (Oryza sativa L.) with the treatment of trinexapac-ethyl and sowing density. Turkish Journal of Agriculture Forestry 3 (37) 257264. doi:10.3906/tar-1207-72.

Unan, R., H. Surek and R. Kaya. 2016. Research on Male Sterility to Commercial Hybrid Rice. Journal of Field Crops Central Research Institute 25 (Special issue-1): 100-104. DOI: $10.21566 /$ tarbitderg. 280269 .

Yuan, L.P. and S.S. Virmani. 1988. Status of hybrid rice research and development. In: Hybrid rice. Manila (Philippines): International Rice Research Institute p 7-24. 